\title{
IMPLANTACIÓN DE UN AULA PILOTO DE HABILIDADES PRÁCTICAS PARA LAS ASIGNATURAS DE PERFIL CLÍNICO DEL GRADO EN VETERINARIA
}

\section{IMPLEMENTATION OF A PILOT CLASSROOM OF PRACTICAL SKILLS FOR THE CLINICAL SUBJECTS OF THE VETERINARY DEGREE}

Carmen Pineda Martos*, Setefilla Quirós Carmona, $\mathrm{M}^{\mathrm{a}}$ del Mar Granados Machuca, Alba Galán Rodríguez

*v32pimac@uco.es

\section{Resumen}

Las limitaciones temporales en la docencia de las asignaturas clínicas del Grado en Veterinaria, así como el elevado ratio estudiantes/profesor y sobre todo estudiantes/paciente, condicionan en gran medida la formación del alumnado, comprometiendo la adquisición de competencias específicas. Así, el alumnado debe amortizar el tiempo que dedica al aprendizaje de estas materias, integrar los conocimientos adquiridos en ellas para llegar a conseguir el fin de las mismas y ser capaz de enfrentarse a situaciones clínicas con la consecuente realización de los necesarios procedimientos manuales. En este artículo, generado a partir de un proyecto de innovación docente, consideramos la enseñanza basada en la práctica con modelos inanimados o maniquíes como imprescindible para complementar la enseñanza de habilidades clínicas y adquisición de competencias.

Debido al alto número de alumnos y al escaso tiempo que pasan por rotaciones clínicas con animales, y teniendo en cuenta las consideraciones éticas, es muy difícil que todos ellos puedan realizar las habilidades clínicas básicas e incluso puedan verlas en situaciones reales. El alumnado debe conocer estos procedimientos dentro de la materia de las asignaturas Propedéutica Clínica, Anestesiología y Cuidados Intensivos, Patología Quirúrgica y Medicina Interna, así como desarrollar estas habilidades, puesto que serán evaluados de esas competencias.

Para ello hemos propuesto la implantación en el Departamento de Medicina y Cirugía Animal de un aula piloto donde los alumnos pueden practicar varias veces en los modelos, con el fin de adquirir la pericia necesaria para el dominio de las habilidades clínicas. De esta forma se fomentará una enseñanza integrativa, que mejorará el aprendizaje y el desarrollo de competencias específicas, además de adquirir otras competencias transversales que ayudarán al futuro veterinario en el ejercicio de su profesión.

Palabras clave: Bienestar animal, Competencias, Simuladores, Veterinaria.

\begin{abstract}
Time limitations in the teaching of clinical subjects of the Veterinary Degree, as well as the high student/teacher ratio and especially student/patient, determine the training of students and compromising the acquisition of specific competences. Thus, students should amortize the time devoted to learning these subjects, integrate the knowledge acquired in them and be able to face clinical situations with the consequent accomplishment of the necessary manual procedures. In the present study, derived from a teaching innovation project, we consider the practicebased teaching with inanimate models or mannequins as a complement of the teaching of clinical skills and acquisition of competences.

Due to the high number of students, the short time they spend in clinical rotations with animals and bearing in mind the ethical considerations, it is very difficult for the students to perform the basic clinical skills and even to see them in real situations. The students must know these procedures within the subjects: Clinical Propedeutics, Anesthesiology and Intensive Care, Surgical Pathology and Internal Medicine, as well as to develop these abilities since they will be evaluated of those competences.

We have proposed the implementation of a pilot classroom in the Department of Animal Medicine and Surgery where students can practice several times in the inanimate models in order to acquire the necessary expertise for the successful development of clinical skills. This initiative will promote an integrative teaching which will improve the learning and development of specific skills, as well as the acquisition of other transversal competences that will help the future veterinarian in the exercise of their profession.
\end{abstract}

Keywords: Animal welfare, Competences, Simulators, Veterinary.

\section{INTRODUCCIÓN}

Las asignaturas de tipo clínico del Grado en Veterinaria se caracterizan por contar con un elevado número de estudiantes, así como por un elevado ratio estudiantes/profesor y sobre todo estudiantes/paciente, lo que limita en gran medida la formación del alumnado, comprometiendo la adquisición de competencias específicas, especialmente en las asignaturas clínicas, en las que estas competencias implican el conocimiento de la materia y la aplicación de éste, además de la adquisición de habilidades prácticas.

A pesar de las mejoras docentes conseguidas, los estudiantes presentan déficits importantes en el aprendizaje, sobre todo, en la consolidación de estas habilidades y procedimientos clínicos básicos, debido a la imposibilidad de consecución de una determinada habilidad en un paciente real. Así, junto con las limitaciones temporales en la docencia de las asignaturas clínicas y el escaso tiempo que pasan los alumnos por rotaciones clínicas, es muy difícil que todos ellos puedan realizar de manera individual procedimientos médicos y quirúrgicos e incluso en ocasiones verlos. El alumno debe conocer y saber 
realizar estos procedimientos considerados básicos dentro de la materia de las asignaturas de Propedéutica Clínica, Anestesiología y Cuidados Intensivos, Patología Quirúrgica y Medicina Interna, puesto que serán evaluados de estas competencias, no sólo durante la docencia teórico-práctica de cada asignatura, sino durante el Prácticum en el que los alumnos se enfrentan a casos reales y completos, en los que deberán integrar los conocimientos y habilidades adquiridas en las diferentes asignaturas clínicas.

Por tanto, es necesario el uso de nuevas herramientas para mejorar el aprendizaje de los estudiantes en el área de las ciencias clínicas, ya que actualmente la docencia teórica se realiza en grupos grandes, y solo algunas prácticas se pueden impartir a grupos considerados pequeños (12 alumnos), pero que son muy grandes en vista a adquirir habilidades y competencias en pacientes reales. En la sociedad actual, y sobre todo para los estudiantes, el aprendizaje de manera práctica supone un mayor impacto y, es en gran medida, la base del aprendizaje. En nuestra área, la práctica y realización individual de técnicas y procedimientos clínicos, frente a la explicación oral y lectura de los mismos, además de reforzar los conocimientos aprendidos en las prácticas de aula, mejora enormemente el aprendizaje y desarrollo posterior de los mismos por el alumnado, formando de esta manera a mejores profesionales.

Teniendo en cuenta lo expuesto anteriormente y que el aprendizaje de determinados procedimientos no puede realizarse sobre pacientes, consideramos el empleo de simuladores como una opción muy interesante para proporcionar a los alumnos la destreza necesaria previa a su actuación directa con el paciente. De hecho, los modelos y simuladores se utilizan cada vez más para complementar la formación práctica de los alumnos de titulaciones de perfil clínico (Baillie et al., 2015; Miller, 1990; Parker et al., 2009; Williams et al., 2006). Aunque en el mercado existe una amplia variedad de simuladores y maniquíes inanimados, éstos superan las posibilidades económicas de los docentes. Es por ello que la nueva tendencia, creada desde las Facultades de Veterinaria de Bristol (Reino Unido) y Hannover (Alemania), aboga por la creación de un aula de habilidades clínicas donde alojar modelos de baja fidelidad que, si bien usando a veces materiales menos sofisticados, llegan a conseguir los mismos objetivos planteados para los simuladores comerciales (Baillie et al., 2015). Este tipo de aula ofrece un ambiente atractivo, seguro y tranquilo, donde los estudiantes pueden practicar varias veces en los modelos, con el fin de adquirir la pericia necesaria para el dominio de las habilidades clínicas. Del mismo modo, este tipo de aula es también un lugar ideal para impartir y desarrollar clases, actividades y evaluaciones de tipo práctico.

Este artículo, fruto de la génesis de un proyecto de innovación docente, propone la ubicación de un aula piloto de habilidades prácticas en el Departamento de Medicina y Cirugía Animal donde los alumnos puedan tener acceso de manera continua a diferentes simuladores en los que aprender y practicar competencias clínicas básicas. De este modo se fomenta una enseñanza integrativa, que favorece el aprendizaje pormenorizado y el desarrollo de competencias específicas, además de adquirir otras competencias transversales que ayudarán al futuro veterinario en el ejercicio de su profesión.

\section{OBJETIVOS}

Los objetivos planteados en el presente artículo se dirigen a asentar los conocimientos prácticos de los alumnos de veterinaria y a implantar un modelo de enseñanza-aprendizaje más fluido que permita asimilar a los estudiantes los procedimientos de manera secuencial, mediante la ubicación de un aula piloto de habilidades clínicas en el Departamento de Medicina y Cirugía Animal para la adquisición de las habilidades de las asignaturas Propedéutica Clínica, Anestesiología y Cuidados Intensivos, Patología Quirúrgica y Medicina Interna, sin redundar en los conocimientos teóricos que el alumno ya posee.

Se han perseguido los siguientes objetivos:

1. Adecuación de un aula piloto de habilidades clínicas para el aprendizaje autónomo y la enseñanza entre iguales, en un ambiente atractivo y accesible que promueva la reflexión de manera conjunta e individual sobre los conocimientos y destrezas aprendidos.

2. Proporcionar un apoyo a la docencia teórico-práctica de diferentes asignaturas clínicas, ya que en muchos casos el desarrollo de las distintas habilidades clínicas está limitado por el número de animales de prácticas o pertenecientes a clientes del Hospital Clínico Veterinario.

3. Permitir a los alumnos realizar los procedimientos de forma segura, disminuyendo el riesgo para el paciente y sin causar daños mayores, permitiendo además al profesor poder explicar cualquier maniobra previo a su realización.

4. Unificar criterios referentes a la práctica clínica entre docentes, ya que existen un número de competencias que pueden ser evaluadas por más de una asignatura durante el transcurso del Prácticum, y que pueden realizarse de diferentes formas siendo todas ellas correctas, lo que puede confundir al alumno a la hora de su aprendizaje. 
Además, de esta forma estas habilidades serían evaluadas con un criterio unificado y justo, ya que todos los estudiantes han recibido la información y la formación práctica necesarias.

\section{Metodología}

Este artículo engloba a diferentes grupos docentes (13, 37 y 126), los cuales son responsables de diferentes asignaturas en el Grado de Veterinaria como Propedéutica Clínica, Anestesiología y Cuidados Intensivos, Patología Quirúrgica y Medicina Interna. El grupo de docentes implicados, además de participar en estas asignaturas, tienen docencia en el Prácticum del Grado de Veterinaria. Desde la explicación-puesta en práctica de una determinada habilidad o competencia durante las prácticas regladas de $3^{\circ}$ y $4^{\circ}$ curso del Grado de Veterinaria, hasta que el alumno realiza el Prácticum en $5^{\circ}$ curso y por tanto tiene la posibilidad de llevarlas a cabo sobre casos reales, tiene a su disposición este aula para poder practicar y recordar todas las destrezas aprendidas en las diferentes asignaturas.

En primer lugar, cada docente seleccionó una o varias actividades de mayor repercusión clínica real, y diseñó una ficha o guía resumen para cada una de las habilidades propuestas, con la finalidad de organizar la información y fomentar el autoaprendizaje. En cada ficha se detalló el procedimiento a realizar y los pasos a seguir "guías paso a paso".

También se incluyeron en esta aula piloto vídeos explicativos de determinados procedimientos clínicos, que fueron maquetados en un proyecto de innovación docente anterior, donde el alumno podía informarse detalladamente de cada habilidad y ver cómo se realiza sobre un modelo real.

Además, cada docente fue responsable de desarrollar o proporcionar un simulador o maniquí sobre el que llevar a cabo las habilidades clínicas seleccionadas. Algunos de estos maniquíes habían sido adquiridos previamente con cargo a otro proyecto docente, en las diferentes áreas del Departamento de Medicina y Cirugía Animal. Por tanto, con el trabajo plasmado en este artículo, se ha conseguido una colaboración y coordinación docente interdisciplinar, además de englobar en esta aula piloto maniquíes y vídeos ilustrativos, así como otros elementos didácticos que han sido adquiridos con proyectos de innovación en años anteriores.

El aula piloto se ha ubicado temporalmente en el Aula Clínica del Hospital Clínico Veterinario (Figura 1). Los distintos modelos inanimados y maniquíes se distribuyeron en diferentes puestos de trabajo categorizados por procedimientos (Figura 2), a la que los estudiantes tuvieron libre acceso durante 4 horas a la semana, repartidas en dos días (2 horas/día) previamente establecidos por la tarde en grupos de 8 estudiantes y convocados con anterioridad (Figura 3). El aula fue de libre acceso a todos los estudiantes de Veterinaria que desearan practicar las habilidades, contando siempre con la supervisión de un profesor que ayudó a los alumnos a llevar a cabo todos los procedimientos de manera correcta sobre modelos o simuladores inanimados (Figura 4).

Las habilidades y destrezas a las que tuvieron acceso los alumnos en esta aula piloto fueron:

1. Técnicas de exploración general.

2. Administración de fármacos vía intramuscular, subcutánea e intravenosa.

3. Extracción de sangre.

4. Cateterización intravenosa.

5. Manejo de catéteres.

6. Cistocentesis.

7. Sondaje uretral.

8. Preparación del campo y del personal quirúrgico.

9. Técnicas básicas de sutura.

10. Intubación endotraqueal.

Además al finalizar cada sesión, se entregó una encuesta para rellenar de manera voluntaria y anónima a cada estudiante que asistió al aula piloto de habilidades, a objeto de recoger su opinión sobre la experiencia y realizar mejoras a tal respecto. Las cuestiones planteadas se evaluaron en escala de 1 a 5 (considerando 1 como nada bueno y 5 como excelente).

\section{RESULTADOS OBTENIDOS}

Se ha creado material didáctico que permite al alumnado la práctica de habilidades clínicas básicas y que consideramos se adecua más correctamente a los objetivos prácticos que se les demanda en su evaluación del Prácticum.

El diseño y organización de las distintas habilidades clínicas englobadas en esta aula piloto ha llevado más tiempo del esperado, por lo que el aula ha estado totalmente acondicionada para su uso durante el segundo cuatrimestre del curso 201617. Una de las razones ha sido el desarrollo de las fichas o guías "paso a paso" anexas a cada puesto de trabajo. Estas guías resumen se desarrollaron durante el mes de octubre y fueron sometidas a un periodo de prueba durante las prácticas regladas de las distintas asignaturas participantes en esta experiencia docente en el primer cuatrimestre del curso $2016-17$. Fueron los propios alumnos de $3^{\circ}$ y $4^{\circ}$ curso los que las valoraron y propusieron ideas de mejora. 
Por otra parte, el objetivo inicial era habilitar una de las salas del sótano del Departamento de Medicina y Cirugía Animal para albergar de manera permanente esta aula piloto. Pero debido a la escasez de espacios acondicionados, y a la falta de financiación para poder habilitar parte de alguno de ellos, finalmente el aula piloto se ubicó en el Aula Clínica del Hospital Clínico Veterinario, la cual tiene una alta demanda durante el primer cuatrimestre con prácticas regladas de las distintas asignaturas clínicas del Grado en Veterinaria, lo que nos llevó a retrasar la fecha de inicio de esta aula piloto. Además, teniendo en cuenta que el desarrollo del aula piloto está enfocado especialmente a estudiantes de $5^{\circ}$ curso, concretamente a la práctica de las distintas habilidades clínicas con vistas a la evaluación del Prácticum, el cual se imparte durante el segundo cuatrimestre.

$\mathrm{Al}$ aula piloto de habilidades prácticas asistió un total de 50 alumnos los cuales realizaron su encuesta de satisfacción tras la realización de las sesiones. El 100\% del alumnado quedó gratamente sorprendido y satisfecho con este tipo de actividad. El poder realizar procedimientos clínicos usando simuladores y modelos inanimados fue percibido con una puntuación media de 4,15 sobre 5 puntos. Los estudiantes valoraron muy positivamente (4,20 sobre 5 puntos) el empleo de modelos inanimados de cara al respeto de los animales de prácticas y al bienestar animal. Sin embargo, consideraron la semejanza de los modelos con la realidad con 3,30 sobre 5 puntos. No obstante, el alumnado se mostró altamente satisfecho (4,90 sobre 5 puntos) ya que pudo reforzar y poner en práctica las destrezas adquiridas y repetirlas cuantas veces fuese necesario. Las guías paso a paso anexas a cada puesto se valoraron con una puntuación de 4,15 sobre 5 puntos. Además, algunos estudiantes pusieron de manifiesto la necesidad de incluir modelos basados en el manejo de animales exóticos e incluso el añadir otras técnicas y habilidades no propuestas en esta experiencia inicial.

\section{UTILIDAD/ANÁLISIS}

Con el desarrollo de esta experiencia docente, los alumnos han incrementado su capacidad de autoaprendizaje y trabajo en grupo, y han adquirido y/o reforzado los conocimientos necesarios para dominar todas la habilidades clínicas incluidas en el aula piloto antes de llevar a cabo estos procedimientos en pacientes reales. Los alumnos reciben todos los conocimientos teóricos básicos para realizar todos estos procedimientos durante las prácticas regladas de los cursos $3^{\circ}$ y $4^{\circ}$ del Grado de Veterinaria. En este aula pueden recordar y practicar todas las habilidades cuantas veces quieran para llegar con suficiente destreza al Prácticum en el $5^{\circ}$ curso, momento en el cual tendrán que realizarlas sobre pacientes reales. Además, creemos que a pesar de ciertas limitaciones, el aula ha sido accesible para los alumnos, y ha motivado su aprendizaje, lo que esperamos redunde en una mejor evaluación en las diferentes asignaturas y en el Prácticum.

Tras la realización del presente trabajo, los docentes implicados han llevado a cabo una evaluación conjunta tanto del funcionamiento como de los resultados obtenidos, obteniéndose una valoración global muy positiva y enriquecedora. Igualmente, mediante la elaboración de las encuestas de evaluación al finalizar las sesiones, se ha valorado el grado de satisfacción de los estudiantes y se han recopilado propuestas de mejora., además los alumnos han valorado positivamente el aula y la mayoría de ellos demandan un mayor número de maniquíes o de puestos de trabajo en los que se puedan practicar otras habilidades a las desarrolladas en el presente estudio.

\section{CONCLUSIONES/DISCUSIÓN}

Consideramos de gran utilidad el poseer este tipo de espacios docentes que suponen un instrumento importante en el aprendizaje y la autoevaluación del alumnado, aunque dicha actividad todavía cuenta con algunas debilidades entre las que se incluyen el obtener financiación para su mantenimineto y el reconocer la actividad llevada a cabo por el profesorado dentro de los Planes Docentes.

La oportunidad de haber podido desarrollar este aula piloto ha supuesto una importante mejora en los recursos docentes de las distintas asignaturas implicadas. El resultado obtenido es el que esperábamos en nuestra propuesta inicial, y aunque ha resultado muy positiva, ha supuesto una importante carga de trabajo para los profesores participantes.

\section{BIBLIOGRAFÍA}

1. Baillie S, Crowther E, Dilly M. The veterinary clinical skills laboratory initiative. REDU: Revista de Docencia Universitaria 2015;13(núm. extraordinario):73-81.

2. Dilly M, Read EK, Baillie S. A survey of established veterinary clinical skills laboratories from Europe and North America: present practices and recent developments. J Vet Med Educ 2017;44(4):580-589.

3. Miller GE. The assessment of clinical skills/competence/performace. Acad Med 1990;65:63-67.

4. Parkes R, Forrest N, Baillies S. A mixed reality simulator for feline abdominal palpation training in veterinary medicine. Stud Health Technol Inform 2009;142:244-246.

5. Williams LE, Nettifee-Osborne JA, Johnson JL. A model for improving student confidence and experience in diagnostic sample collection and interpretation. J Vet Med Educ 2006;33(1):132-139. 


\section{LEYENDAS DE FIGURAS}

Figura 1. Vista del aula piloto de habilidades clínicas.

Figura 2. Vista de algunos de los maniquíes o simuladores empleados en el desarrollo de esta experiencia docente. Figura 3. Grupos de estudiantes en el aula piloto.

Figura 4. Supervisión y ayuda continua del profesorado.

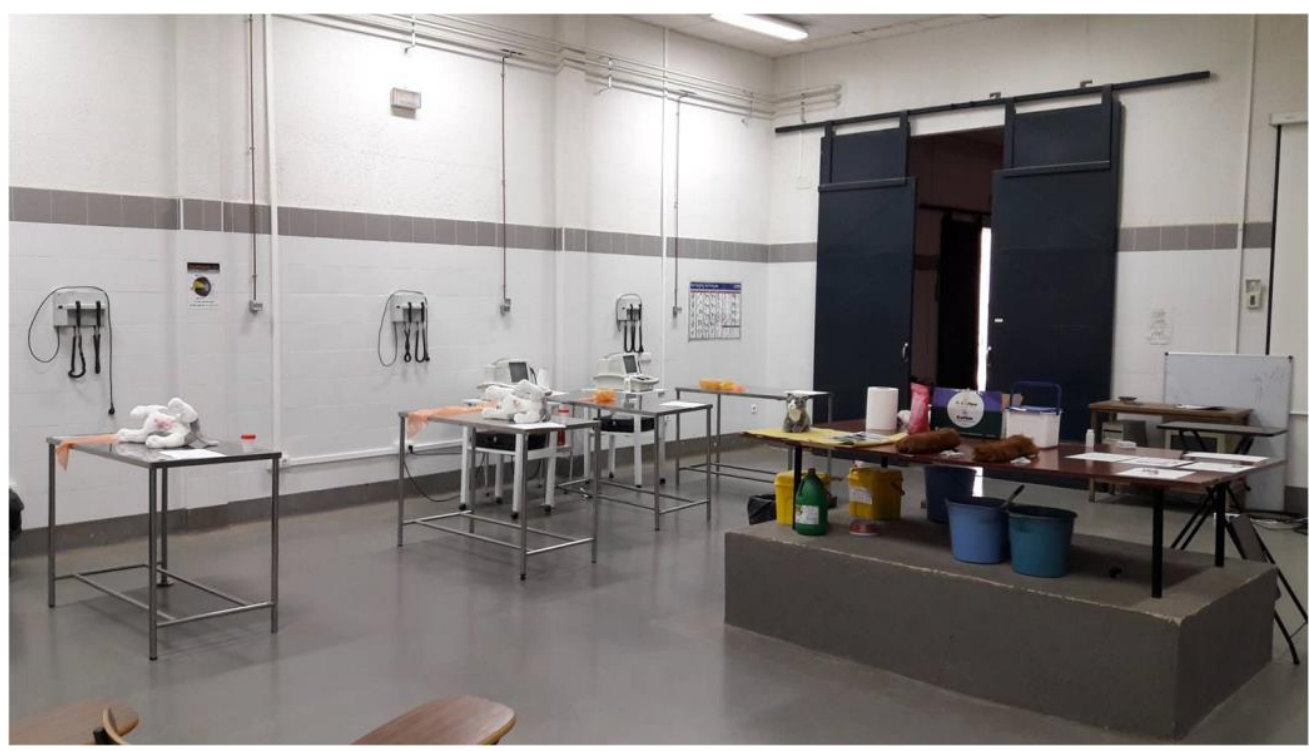

Figura 1. Vista del aula piloto de habilidades clínicas.

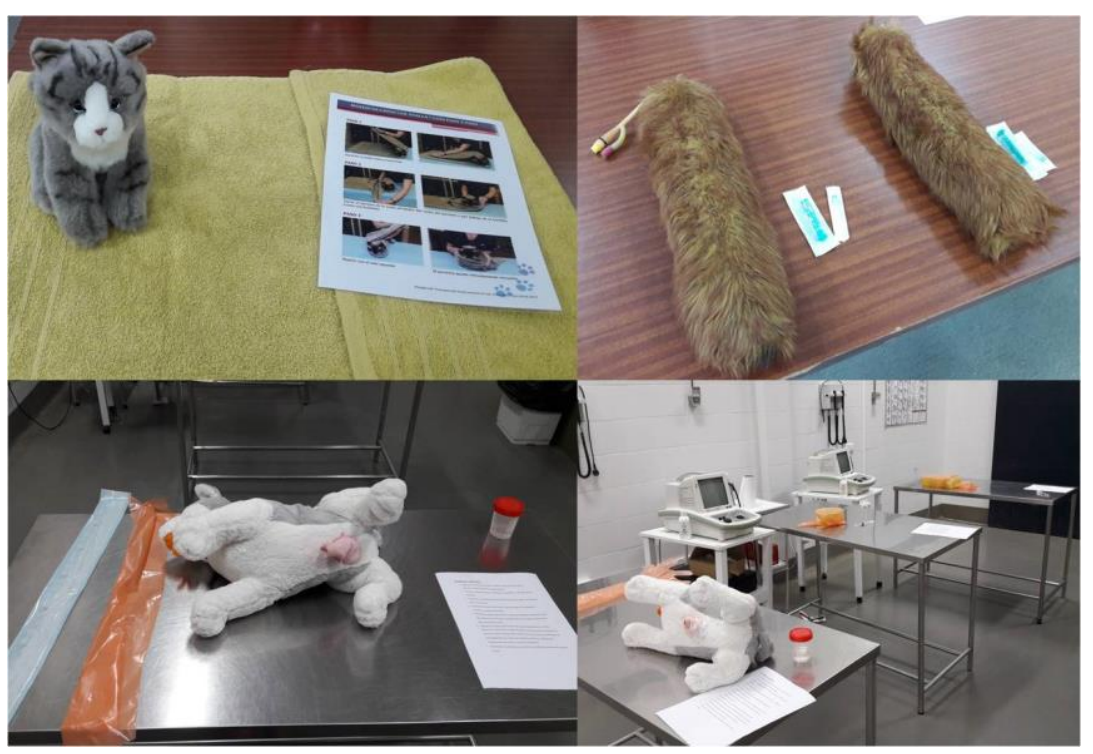

Figura 2. Vista de algunos de los maniquíes o simuladores empleados en el desarrollo de esta experiencia docente. 


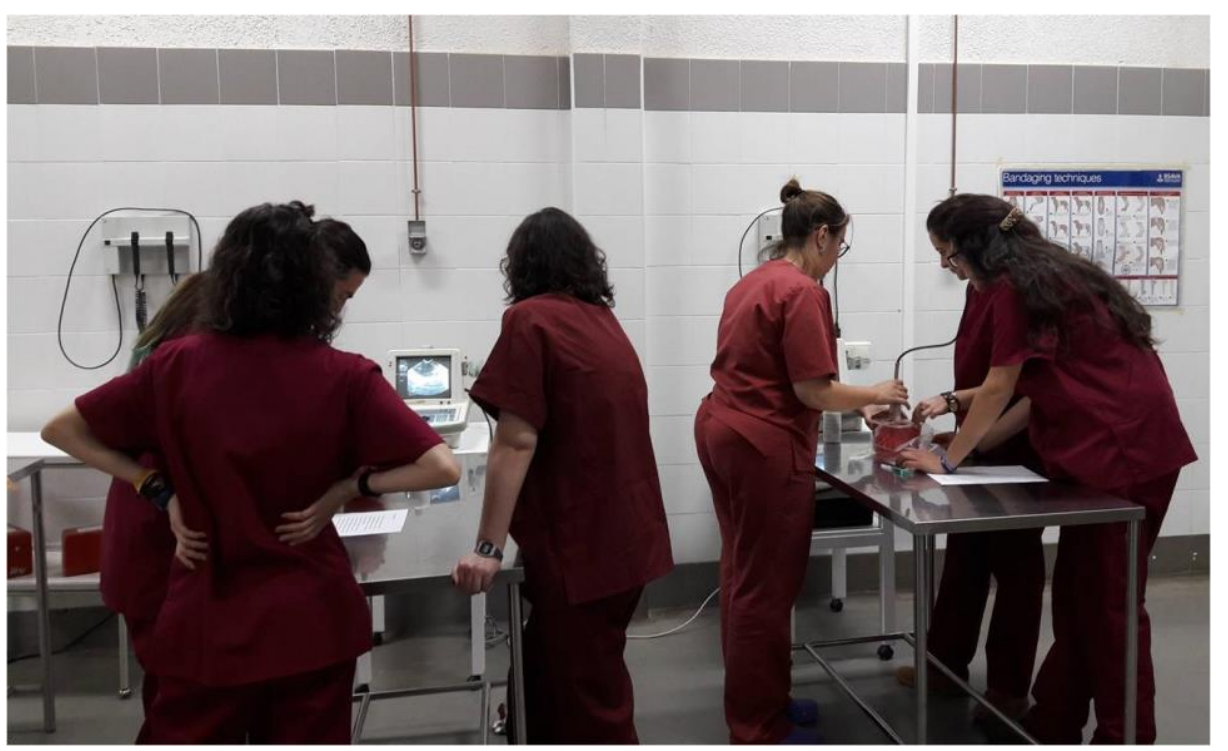

Figura 3. Grupos de estudiantes en el aula piloto.

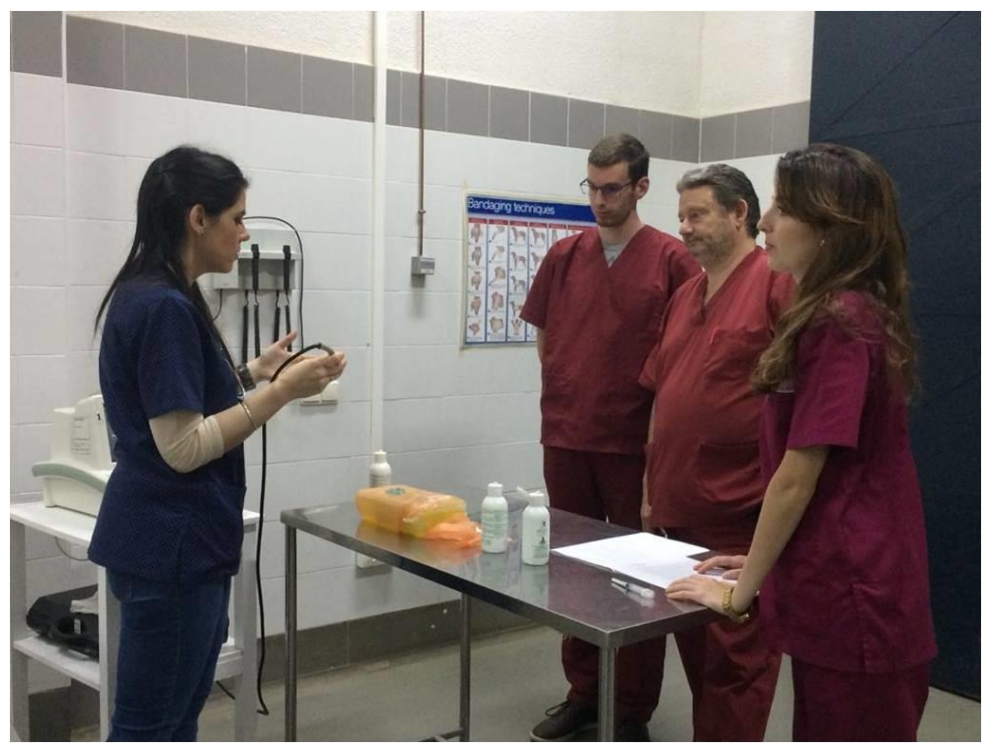

Figura 4. Supervisión y ayuda continua del profesorado. 